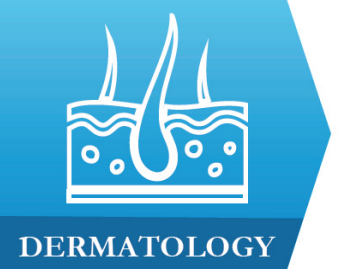

1) Department of General Surgery, Kasturba Medical College, Manipal Academy of Higher Education, Manipal, India

2) Department of Pathology, Kasturba Medical College, Manipal Academy of Higher Education, Manipal, India

\title{
Generalized hypertrophic lichen planus: an atypical presentation of a characteristic disease
}

\author{
Sharan Mirchandani ${ }^{1}$, Devesh Sanjeev Ballal ${ }^{1}$, Gabriel Rodrigues ${ }^{1}$, \\ Kanthilatha $\mathrm{Pai}^{2}$
}

\begin{abstract}
Lichen planus is a common chronic inflammatory condition that affects skin and mucous membranes. Management is often delayed because of patient embarrassment or misdiagnosis by the clinician. Early recognition and treatment is essential to reduce the morbidity of this condition. We present a 58-year-old woman diagnosed with generalized hypertrophic lichen planus, a rare event, reported to occur usually on lower extremities, but found to be generalized in our patient, requiring excision of symptomatic lesions. We suggest a multidisciplinary approach to allay the anxiety and symptoms of these patients and to improve the quality of life and clinical outcomes.
\end{abstract}

Keywords: lichen planus, inflammation, pruritis, excision, steroids, recurrence

\section{Introduction}

Lichen planus (LP) is an uncommon disorder that usually affects middle aged adults and is more frequent in women. It may affect the skin, oral cavity, genitalia, scalp, nails or esophagus. Cutaneous LP occurs in less than $1 \%$ of the population [1]. Variants of LP include hypertrophic, annular, bullous, actinic, LP pigmentosus, inverse, atrophic and follicular. Hypertrophic LP is typified by intensely pruritic, flat-topped plaques that usually involve the lower limbs [2]. The diagnosis of LP is usually clinical, however in certain atypical cases a skin biopsy is required to confirm the diagnosis. Due to the varied presentation and large number of similar appearing conditions, recognition is often delayed.

\section{Case Report}

A 58-year-old female with no DOI: $10.15386 / \mathrm{mpr}-1383$

Manuscript received: 05.06.2019

Received in revised form: 08.10.2019

Accepted: 12.01.2020

Address for correspondence:

gabyrodricks@gmail.com

This work is licensed under a Creative Commons Attribution-NonCommercialNoDerivatives 4.0 International License comorbidities, presented with complaints of itchy lesions over both upper and lower limbs of 2 years duration. At the start, the lesions had appeared over the right foot, ascended upwards to involve the whole of the limb and then were seen on the left lower limb and over both upper limbs. The lesions were papulesin the beginnig, then progressed to multiple pruritic warty lesions and were non-discharging.
There was no other contributory history. On examination, the majority of the lesions were on the extremities and were tender, multiple, well-defined, hypo and hyperpigmented, hyperkeratotic, rough, verrucous-like, scaly papules, plaques and nodules (Figure 1). Few of them were excoriated with crusting predominantly seen on dorsal arms and forearms. Similar lesions were also found over the neck, left shoulder, trunk, mid and lower back. A differential diagnosis of generalized LP and systemic lupus erythematosus (SLE) was made. An excision of the lesion on the foot was done of which histopathology showed epidermis with hyperkeratosis, irregular acanthosis, pseudoepitheliomatous hyperplasia, discontinued hypergranulosis, and focal parakeratosis. Underlying dermis showed dense infiltrate composed of lymphocytes, eosinophils, few plasma cells and neutrophils, histiocytes forming Max Joseph spaces due to hydropic degeneration of basal cells, melanin incontinence and numerous proliferating blood vessels, which were characteristic of hypertrophic LP (Figure 2). A direct immunofluorescence study was also done to rule out SLE and was found to be negative. She was initially treated with topical steroids and emollients, with flattening of the lesions over the upper limbs and trunk. 

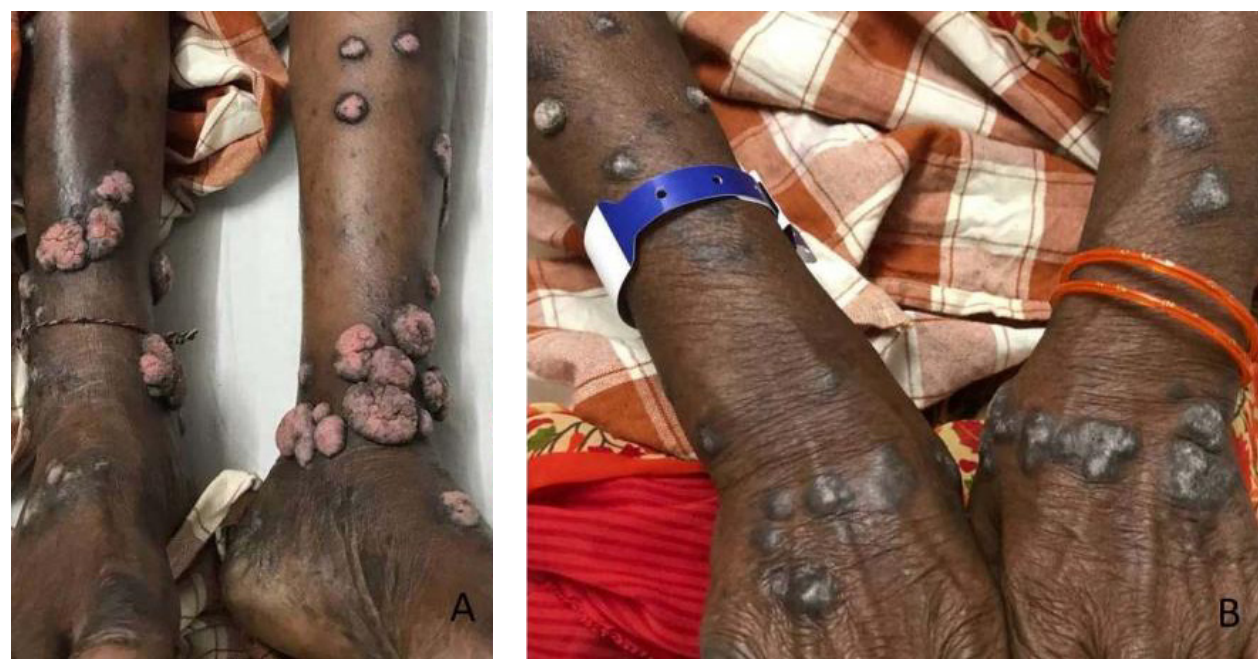

Figure 1 (A \& B). Multiple, well-defined, discrete, hyperpigmented, hyperkeratotic, scaly lesions on the extremities.

The lesions over the lower legs did not respond to topical steroids and underwent excision of multiple lesions, as per her request. As she was depressed due to the generalized disease, counseling was initiated. She was seen after 3 months with lesions appearing flat, not flaring up, no new lesions and pain free.

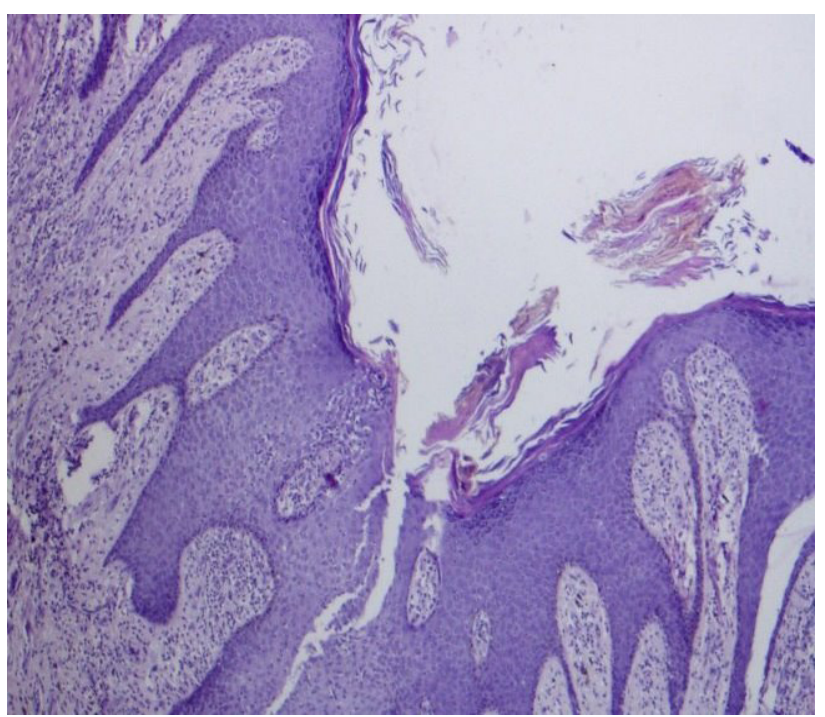

Figure 2. Photomicrograph showing hyperkeratosis, irregular acanthosis, pseudoepitheliomatous hyperplasia, wedge shaped hypergranulosis, focal parakeratosis with a dense lymphocytic and eosinophilic infiltrate ( $\mathrm{H}$ and $\mathrm{E}, \mathrm{x} 40)$.

\section{Discussion}

LP is an idiopathic disorder which is thought to be mediated by an immune-mediated process that involves activated $\mathrm{T}$ cells, mounting an immune response against keratinocytes [3]. Increased levels of ICAM-1 (intercellular adhesion molecule-1) and Th1 related cytokines (TNF- $\alpha$, IFN- $\gamma$, etc.) may also play a role [4]. There is also a relationship between Hepatitis $\mathrm{C}$ virus (HCV) infection and LP, though a causal relationship has not been proven. Patients with $\mathrm{HCV}$ infection are more likely to develop LP than controls [Odds Ratio (OR) 2.5, 95\% Confidence Interval (CI), 2.0-3.1] [5]. Moreover, drug eruptions that closely resemble LP are well documented with numerous antimicrobials such as isoniazid (INH), griseofulvin, ketoconazole, etc., H2 blockers, antihypertensives such as Angiotensin-converting enzyme (ACE) inhibitors, beta blockers, prazosin), antimalarials, diuretics and gold salts [6].

The characteristic appearance of cutaneous LP is a papulosquamous rash with flat-topped, violaceous papules in the skin. The clinical features have been described as the 4 P's: pruritic, purple, polygonal, plaques. Individual papules are generally only a few millimeters but may fuse leading to larger plaques [7]. Faint white lines can be seen of these papules/plaques and eponymously named "Wickham's Striae". LP may also demonstrate the Koebner reaction, wherein lesions develop at sites of trauma (most often due to scratching) and usually heal with significant hyperpigmentation [2-5].

LP is usually a clinical diagnosis and has pathognomonic pathologic features that aid in the diagnosis of atypical cases: hyperkeratosis and no parakeratosis; vacuolization in basal layer of epidermis; Civatte bodies; saw-tooth shaped rete ridges; Max Joseph spaces; bandlike lymphocyte infiltration at the junction of dermis and epidermis; eosinophilic inclusion bodies in the papillary dermis and pigment changes that are more evident in dark skinned patients [8].

The key differentials that need to be borne in mind when diagnosing LP are lichenoid drug eruptions 
and chronic graft-versus-host disease, both of which can be excluded with a thorough history $[1,2]$. Other papulosquamous lesions like psoriasis, lichen simplex chronicus, SLE, pityriasis rosea and syphilis may be confused for LP, but a careful examination can exclude most of these conditions [2,3].

The natural history of cutaneous LP is to remit within one to two years, while lesions affecting the scalp, genitals and nails may be more persistent [9]. Since this disorder is self-resolving, management is usually focused speeding up the process of resolution and treating pruritis. Topical corticosteroids are first line medications and patients should be warned about cutaneous atrophy that occurs with their use [10]. Thicker lesions may be tackled with intralesional corticosteroids that is particularly beneficial in hypertrophic LP, as in our patient. Hypopigmentation and cutaneous atrophy are documented side effects and should be looked for [11]. Second line therapy with oral steroids, phototherapy or oral acitretin is reserved for patients with topical-steroid refractory disease or in generalized disease where topical therapy is not feasible $[4,11]$. A multidisciplinary team comprising a dermatologist, immunologist, surgeon, and a psychologist is required to manage these patients.

\section{References}

1. Puza C, Cardones AR. Concepts and controversies in the treatment of cutaneous lichen planus. G Ital Dermatol Venereol. 2017;152:607-614.

2. Atzmony L, Reiter O, Hodak E, Gdalevich M, Mimouni D. Treatments for cutaneous lichen planus: A systematic review and meta-analysis. Am J Clin Dermatol. 2016;17:11-22.

3. Fazel N. Cutaneous lichen planus: A systematic review of treatments. J Dermatolog Treat. 2015;26:280-283.

4. Humberto JSM, Pavanin JV, Rocha MJAD, Motta ACF. Cytokines, cortisol, and nitric oxide as salivary biomarkers in oral lichen planus: a systematic review. Braz Oral Res. 2018;32:e82.

5. Das A, Panda S. Use of topical corticosteroids in dermatology: An evidence-based approach. Indian J Dermatol. 2017;62:237-250.

6. Alaizari NA, Al-Maweri SA, Al-Shamiri HM, Tarakji B, Shugaa-Addin B. Hepatitis C virus infections in oral lichen planus: a systematic review and meta-analysis. Aust Dent J. 2016;61:282-287.

7. Yousefi H, Abdollahi M. An update on drug-induced oral reactions. J Pharm Pharm Sci. 2018;21:171-183.

8. Cheng YS, Gould A, Kurago Z, Fantasia J, Muller S. Diagnosis of oral lichen planus: a position paper of the American Academy of Oral and Maxillofacial Pathology. Oral Surg Oral Med Oral Pathol Oral Radiol. 2016;122:332354.

9. Thandar Y, Maharajh R, Haffejee F, Mosam A. Treatment of cutaneous lichen planus (part 2): a review of systemic therapies. J Dermatolog Treat. 2019;30:633-647.

10. Ferri EP, Gallo CB, Abboud CS, Yanaguizawa WH, Horliana ACRT, Silva DFTD, et al. Efficacy of photobiomodulation on oral lichen planus: a protocol study for a doubleblind, randomised controlled clinical trial. BMJ Open. 2018;8:e24083.

11. Totonchy MB, Leventhal JS, Ko CJ, Leffell DJ. Hypertrophic lichen planus and well-differentiated squamous cell carcinoma: a diagnostic conundrum. Dermatol Surg. 2018;44:1466-1470. 